\title{
derniers développements dans le clouage des sols
}

\author{
latest developments in soil nailing
}

\author{
C. LOUIS \\ Claude Louis, Constructions S.A. *
}

\section{Résumé}

Le renforcement des sols en déblais par clouage connaît actuellement un grand développement. Des réalisations de plus en plus audacieuses sont entreprises et les techniques évoluent considérablement de manière à améliorer les performances et la pérennité des éléments intervenant dans la méthode, à savoir : peau de surface, ancrages, drainage, bourrage et consolidation.

Des systèmes très performants ont été mis au point à ce sujet pour la réalisation de la peau de surface à l'aide d'éléments préfabriqués assemblés entre eux. De même, en particulier dans les terrains difficiles, une amélioration des techniques de mise en œuvre d'ancrages est à signaler avec des clous de grande longueur, vibrofonçables dans les terrains instables avec éventuellement préinjection, voire traitement avant, pendant et après la mise en œuvre des clous.

Les dernières réalisations récentes sont présentées.

\section{Abstract}

The reinforcement of soil slopes in cutting by nailing is undergoing a rapid development. Projects undertaken have become more and more daring while the techniques involved have developed considerably in a manner so as to improve the performance and the durability of the various elements involved in the process, namely: face covering, anchoring, drainage, backfilling and backgrouting.

With regard to the face covering, highly successful results have been obtained by the use of a system of interlocking precast elements. Similarly, particularly in difficult ground conditions, a number of advancements have been made in the method of installing the anchorages with the development of techniques using vibration for placing nails of great length in unstable ground, with the possibility of pregrouting, or general ground-treatment in advance, during or after the installation of the nails.

Examples of some recent projects are shown.

\footnotetext{
- 12, avenue Franklin-Roosevelt, 75008 Paris.
} 


\section{POSITION DU PROBLÈME}

Le renforcement des sols et des roches, en particulier pour le soutènement d'excavations par la technique de clouage (soil nailing), a connu au cours de la présente décennie un développement très important dans divers pays. Cette technique consiste à mettre en place dans le terrain, soit par l'intermédiaire de forages, soit par vibrofonçage, des inclusions métalliques (ou autres matériaux de grande résistance) pour améliorer les caractéristiques mécaniques globales du milieu composite ainsi constitué. Ces inclusions sont généralement scellées au terrain pour améliorer leur interaction avec le sol et donc leur efficacité. Dans de nombreux cas, les inclusions émergent à l'extérieur de la surface de l'excavation et sont reliées entre elles par une peau résistante, souple, dite a peau de confinement ".

Les principes de cette méthode de soutènement ainsi que des recommandations pour sa mise en ceuvre ont été exposés en détail dans une publication antérieure de l'auteur (LOUIS, 1981). Ils s'apparentent tout à fait à ceux quil interviennent dans la nouvelle méthode autrichienne de construction des tunnels et l'on peut considérer que la technique du clouage est une extension, pour les problèmes à ciel ouvert, de ladite méthode autrichienne.

Le but du présent article vise en premier lieu à faire le point sur les développements récents de cette technique du clouage des sols. Des progrès ont certes été réalisés mais un travail important reste cependant encore à faire pour ce qui concerne la justification théorique des ouvrages soutenus par clouage. Par ailleurs, les méthodes de mesures relatives à cette technique ne sont pas encore suffisamment développées dans la pratique. L'expérience montre hélas que lors d'applications pratiques, les mesures les plus élémentaires sont négligées alors qu'elles jouent un rôle primordial dans une approche correcte du dimensionnement. Ces mesures concernent essentiellement P'interaction solinclusions.

A l'origine, la peau de confinement en surface était essentiellement basée sur l'utilisation du béton projeté. Cette technique reste tout à fait intéressante quant à son efficacité mais présente par contre quelques inconvénients sur le plan fonctionnel et surtout pour sa pérennité. Cela est d'autant plus vrai pour les ouvrages définitifs soumis à des conditions d'environnement difficiles. Par ailleurs, la projection du béton rend délicate la mise en œuvre d'un drainage généralisé efficace au contact béton-terrain.

Dans ce contexte, une présentation des derniers développements technologiques est faite dans le présent article de manière à mettre en évidence la grande variété de matériaux et de matériels disponibles actuellement, pour améliorer l'efficacité du système, tout en permettant une réduction notable des coûts de mise en œuvre.

Il serait très long de faire une présentation des références bibliographiques relatives à cette technique, d'autant plus que de nombreuses applications intéressantes n'ont pas été l'objet de publications. Des développements conjoints, souvent indépendants, ont vu le jour en Amérique (Nord et Sud), au Japon, en Europe et en particulier en Allemagne Fédérale (STOCKER, 1976; GAESSLER, 1978 et $1984 \ldots)$ et aussi en France (RABEJAC et al., 1974; SCHLOSSER et al., 1979, 1983; LOUIS, 1971; JURAN et al., 1981 CARTIER et GIGAN, 1982 ; etc.).

Par ailleurs, un séminaire de l'École Nationale des Ponts et Chaussées à Paris (19-22 octobre 1982) sur l'amélioration des sols et des roches par renforcement a été particulièrement orienté vers ce sujet.

\section{RAPPEL DES PRINCIPES DE LA MÉTHODE}

En vue de présenter de manière synthétique les principes de la méthode, il importe de distinguer clairement les différents types de clouage généralement utilisés. Cette distinction doit être faite eu égard aux divers modes de sollicitation des inclusions qui peuvent travailler selon trois modes bien distincts, à savoir :
a) traction-cisaillement,
b) cisaillement pur,
c) compression-cisaillement,
comme le montre schématiquement la figure 1.
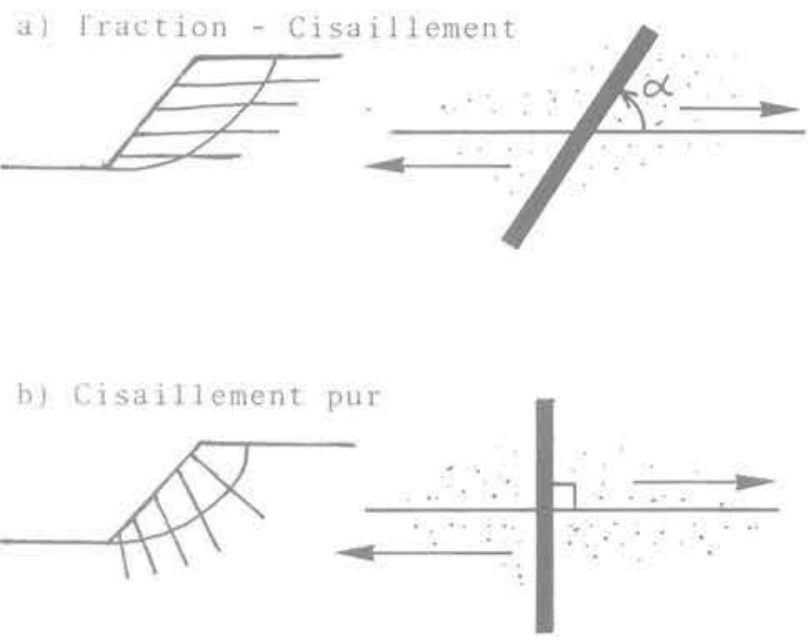

c) Compression cisaillement

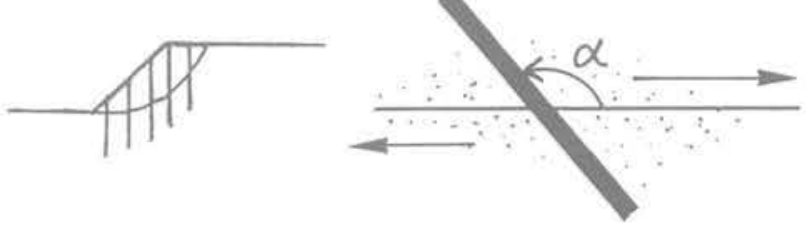

Fig. 1. - Schéma du mode de sollicitation d'une inclusion s'opposant à une rupture par cisaillement ( $b$ et $c$ : " DOVEL PILES ». 
Dans cette différenciation, une remarque importante s'impose concernant la mise en ceuvre de ces inclusions. Deux cas fondamentalement différents doivent être envisagés :

- la mise en cuvre du clouage à l'avancement à l'occasion d'une excavation par passes élémentaires (fig. 2).

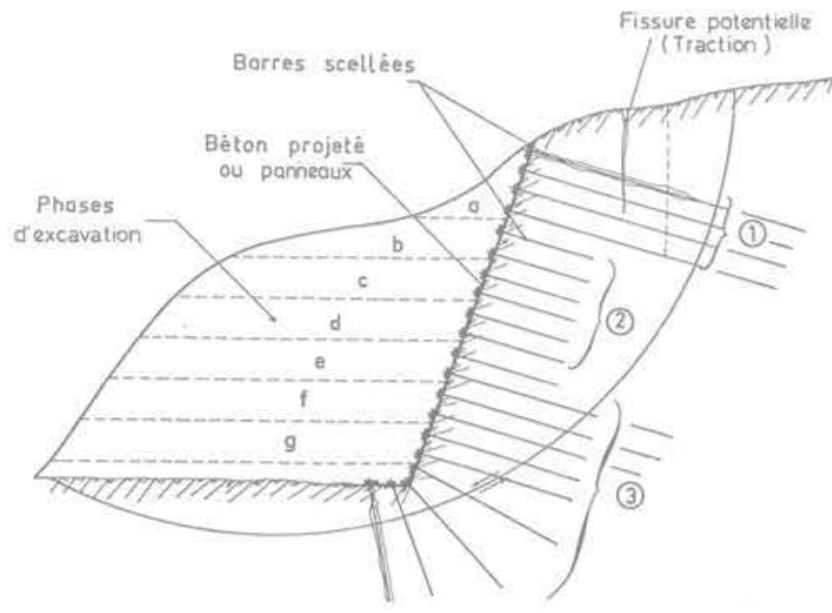

Fig. 2. - Clouage à l'avancement en déblais.

(1) Barres en traction. - (2) Barres intermédiaires.

(3) Barres en cisaillement.

L'excavation joue un rôle capital dans le procédé. L'originalité du procédé réside dans le fait que les inclusions et la peau de confinement sont mises en ceuvre dans les phases initiales de travaux avec une géométrie (type, longueur, densité, etc.) conforme au stade définitif de l'excavation. Les déplacements et la redistribution des contraintes dans le terrain au cours des phases d'abattage successives contribuent à une mise en tension stabilisatrice des inclusions, ceci en fonction du besoin du terrain.

- la stabilisation préventive ou curative de talus ou remblais préexistants généralement en mouvement par des profilés rigides s'opposant aux déplacements (par exemple fig. 1 b et c).

Dans ce cas, les profilés ont des inerties beaucoup plus importantes. Ils sont généralement placés verticalement et s'opposent, par leur rigidité, aux déplacements du terrain. Cette méthode a été en particulier utilisée en France de longue date avec succès par la S.N.C.F, (VERRIER et al., 1981). Dans ce cas, le mode de sollicitation des inclusions est tout à fait différent de celui intervenant dans le cas précédent. C'est ici le développement de poussées-butées par le sol en mouvement qui entraîne une réduction, voire une stabilisation des déplacements. Ce mode de soutènement n'est pas abordé dans le présent article.

La figure 3 permet de mieux comprendre, de manière illustrée, le principe de la méthode du clouage mise en cuvre lors d'excavations, en comparaison avec les inconvénients liés par exemple à l'exécution d'un mur

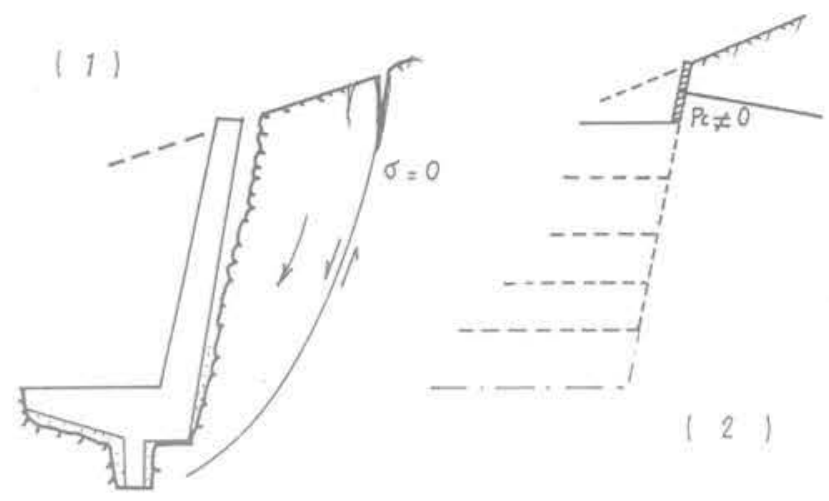

Fig. 3. - Mur de soutènement classique (1) et rupture potentielle en cours de travaux, comparés à une stabilisation à l'avancement par clouage (2).

de soutènement traditionnel « mur poids " (fig. $3 \mathrm{a}$ ). Le principe fondamental retenu vise à perturber au minimum, lors des travaux, le terrain en place, de manière à profiter au maximum de ses caractéristiques mécaniques inititales. La sauvegarde de ces caractéristiques est obtenue en luttant contre certains états de contraintes très néfastes (en particulier état de contrainte uniaxiale) et également contre les déplacements exces sifs. La peau de surface mise en œuvre à l'avancement apporte un minimum de confinement et les inclusions scellées au terrain s'opposent au développement des déformations. Le processus mécanique qui intervient est schématisé de manière comparative sur la figure 4 qui donne, dans la représentation de COULOMB, la schématisation des ruptures lors de la mise en cuvre d'un mur poids (avec excavation non soutenue dans la phase travaux sur une grande hauteur) et l'effet stabili-

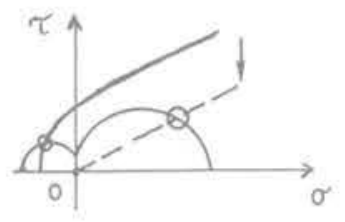

(1)

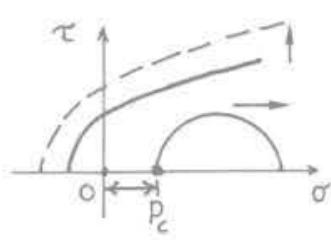

(2)
(3)

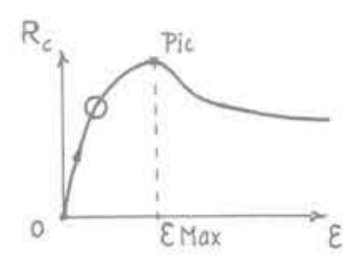

Fig. 4. - Schématisation des avantages sur le plan mécanique de la méthode du clouage.

(1) Elimination des efforts néfastes de la décompression.

(2) Consolidation en peau et dans le massif et développement d'une pression de confinement stabilisatrice.

(3) Limitation des déformations et sauvegarde des caractéristiques mécaniques initiales du terrain. 
sateur du confinement dans la méthode du clouage. La courbe contrainte-déformation montre qu'avec un tel soutènement par clouage à l'avancement, il devient possible de rester toujours en deçà du «pic » dans la courbe effort-déformation du terrain (fig. $4 c$ ), au-delà du «pic» pour des grandes déformations, seule une résistance résiduelle beaucoup plus faible reste disponible:

Dans le cas d'excavations sans soutènement à l'avancement, des ruptures inévitables se produisent en raison de la décompression du massif, avec dans certains cas, action du "mort terrain " sans résistance ou presque, agissant par leur poids sur la structure en fin de travaux, d'où le surdimensionnement nécessaire pour de telles structures. Un processus tout à fait similaire intervient dans les travaux en souterrain.

\section{DIMENSIONNEMENT}

Le dimensionnement d'excavations à ciel ouvert soutenues par clouage a été l'objet de nombreuses publications, en particulier SCHLOSSER et autres, 1979 et 1981; GAESSLER, 1982; JURAN, 1977 et 1985; SHEN, 1981; JEWELL, 1980; LOUIS, 1981. Le principe repose sur l'analyse de trois conditions de stabilité, à savoir :

\section{Stabilité du volume monolithique du terrain armé}

Cela inclut l'examen des risques de glissement sur la base du volume de terrain armé et également des risques de basculement autour du centre instantané de rotation, ceci sous l'effet des sollicitations externes appliquées au volume armé, ce dernier agissant par son propre poids.

\section{Stabilité interne du volume avec des ruptures potentielles traversant le volume armé}

\section{Stabilité en grand pour des ruptures externes}

Autant les conditions un et trois évoquées ci-dessus connaissent une approche théorique satisfaisante, autant l'analyse de la stabilité interne est encore actuel. lement sujette à caution. En effet, dans la plupart des cas, les analyses de stabilité sont menées à la rupture par des méthodes de calcul analogues à celles développées en mécanique des sols pour la stabilité des pentes (méthodes de FELLENIUS, BISHOP ou autres).

Des programmes de calcul automatiques sur ordinateur ont été mis au point. Ils tiennent compte de ruptures internes quelconques avec prise en compte de la présence des inclusions.

Le long de lignes de ruptures potentielles, il est supposé que les armatures rencontrées travaillent de manière uniforme avec des tensions stabilisatrices supposées proportionnelles à la longueur d'ancrages scellés dans le terrain au-delà de la ligne de rupture (fig. 5). Ceci est appliqué pour toutes les lignes de ruptures possibles.

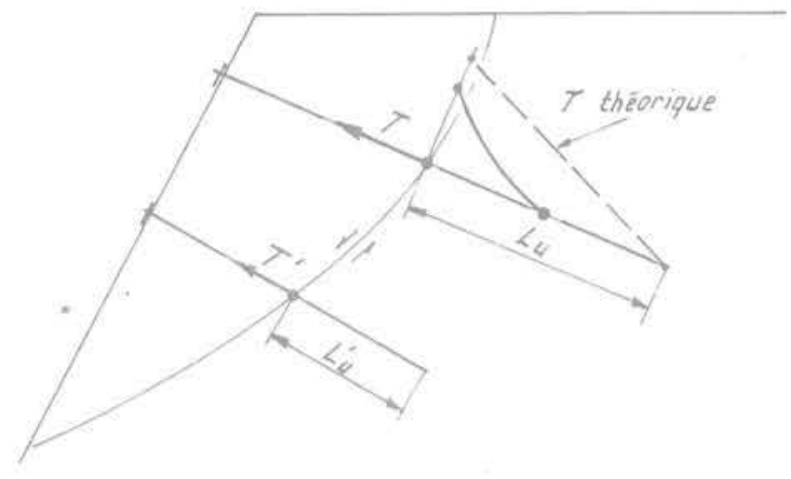

Fig. 5, - Tension stabilisatrice dans l'inclusion supposée proportionnelle à la longueur utile.

Dans une telle analyse, on suppose donc que toutes les armatures travaillent de la même façon, de manière homogène, et qu'elles agissent simultanément. Or, dans la pratique, la répartition des tensions dans les armatures reste inconnue, voire aléatoire. Ces tensions dépendent de la géométrie du massif et surtout de ses caractéristiques géotechniques (dans le cas présent, les hétérogénéités jouent un rôle considérable dans leur répartition). De plus, elles varient notablement dans le temps. L'examen à posteriori de ruptures réelles a montré que la mise en tension des armatures était très hétérogène et que les ruptures étaient progressives. Quelques scellements cèdent par excès de contraintes ou quelques armatures se rompent entraînant de proche en proche des ruptures locales, puis généralisées, du massif (ex. : rupture du talus cloué des Eparris où seul le lit supérieur des armatures en tension s'est rompu au droit de rallonges défectueuses situées à $1,50 \mathrm{~m}$ de profondeur. Cette rupture du premier lit d'ancrages a entraîné une instabilité d'ensemble, sans rupture des autres armatures. Les armatures intermédiaires ont glissé à l'extérieur du scellement, les armatures de pied n'ayant par contre pas été sursollicitées).

Une approche plus correcte du dimensionnement consisterait à déterminer la mise en tension réelle des inclusions. Sur le plan pratique, cela nécessite de faire une étude des déplacements et contraintes, par exemple par la méthode des éléments finis, en tenant compte non seulement des paramètres géomécaniques du milieu, mais également de la présence des armatures (délicates à simuler). La connaissance des tensions dans tout le volume permettrait ensuite de faire une analyse correcte de la stabilité.

Les méthodes de calcul évoquées ci-dessus font appel à une connaissance du cisaillement maximum admissible au contact scellement-terrain. Ce paramètre ne peut être estimé que par des essais in situ appropriés, par exemple par des essais d'arrachement tels que représentés sur la figure 6 . A titre indicatif, la résistance à l'arrachement par unité de longueur peut varier facilement de 1 à 10 , voire de 1 à 20 , dans les sols meubles. Cette résistance est très faible pour les sols à tendance plastique (faible angle de frottement, indice de plasticité élevé), par exemple: argile de Francfort, marne d'Argenteuil, ... Elle est par contre très élevée 


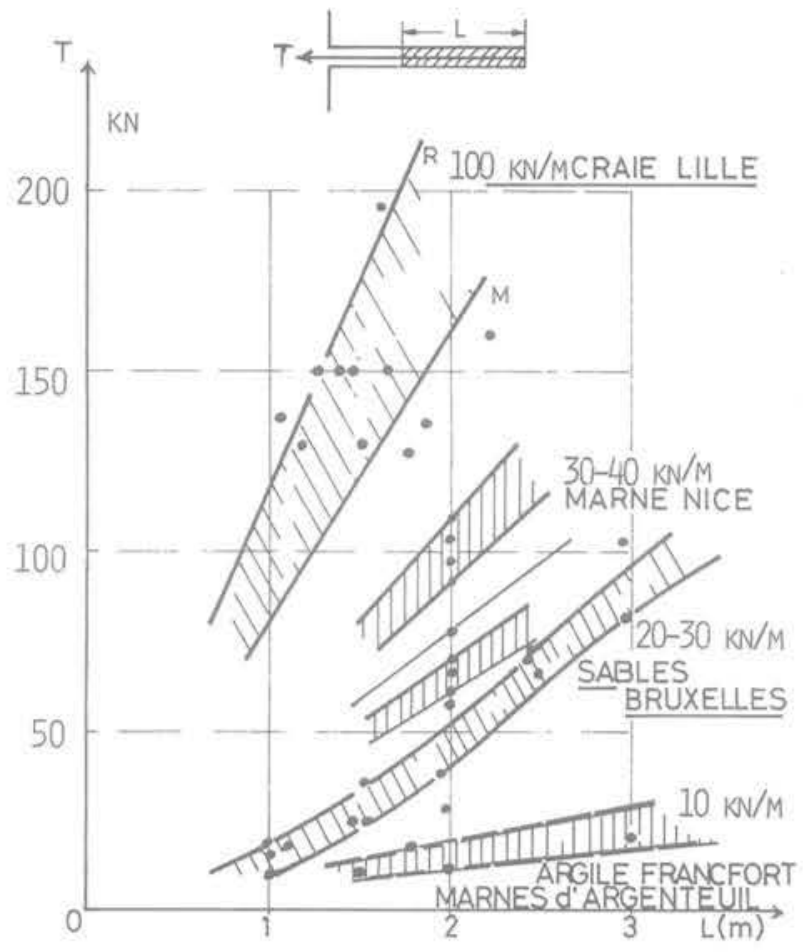

Fig. 6. - Résistance à l'arrachement de boulons à ancrage réparti selon la longueur de scellement (résine ou mortier) dans différents sols (Ø forage 35 à $55 \mathrm{~mm}$ )

dans les sols dilatants (sols compacts granulaires à fort angle de frottement, alluvions sablo-graveleuses compactes, conglomérats, etc.). Cette résistance au cisaillement le long d'armatures dépend en effet surtout de la nature du sol, de la surface latérale du scellement, mais également de la technique de mise en œuvre de l'armature. Elle peut être considérablement accrue par la mise en cuvre de certains vibrofonçages avec injection après, ou mieux pendant le vribrofonçage (technique de CLOU JETON, JET BOLT, voir ci-après).

Étant donné le rôle considérable joué par les inclusions scellées au terrain dans le processus de stabilisation par clouage, il est capital, au stade actuel d'avancement de la technique, d'entreprendre des campagnes de mesures précises sur le comportement de ces dernières, suite à une mise en tension naturelle ou artificielle. Il apparaît en effet que :

- la loi de répartition des efforts dans les boulons est rarement linéaire au-delà d'une zone de mise en tension, en tête ou au milieu du boulon;

- une augmentation de la longueur du boulon n'entraîne pas pour autant un accroissement de sa capacité de résister à l'arrachement ;

- il existe une force critique limite qui ne peut être dépassée. Cette résistance limite est donnée, soit par la résistance du scellement au-delà duquel un fluage du terrain intervient, soit par la résistance de l'acier dans le cas de scellements en terrains très compétents. La figure 7 illustre les idées évoquées ci-dessus en mon- trant les limites du procédé, par exemple par transfert des efforts vers l'intérieur du massif en cas de dépassement de la capacité de résistance du contact terrainscellement. Dans la pratique, pour le bon dimensionnement d'un clouage, il conviendrait de ne mobiliser que la moitié, voire les deux tiers maximum de la résistance au cisaillement du contact terrain-scellement.
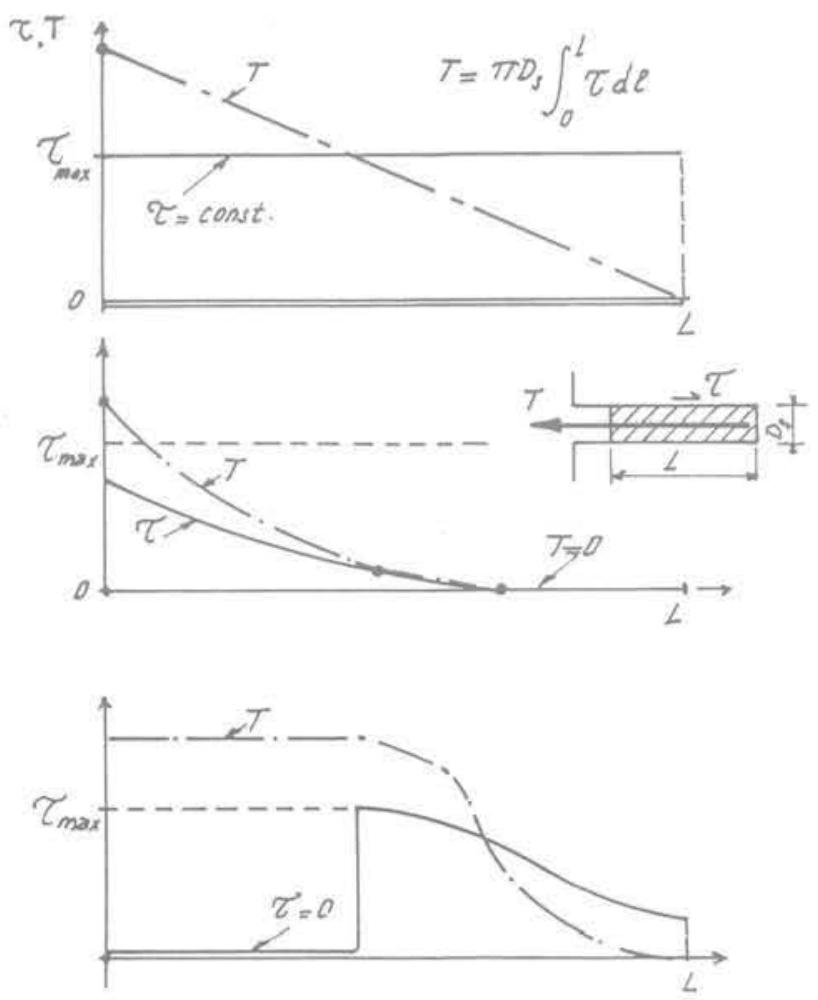

Fig. 7. - Répartitions possibles des efforts lors d'essais de traction sur armatures scellées.

En complément, la figure 8 donne deux répartitions d'efforts le long des boulons en cas de mise en charge instantanée du scellement à la tête.

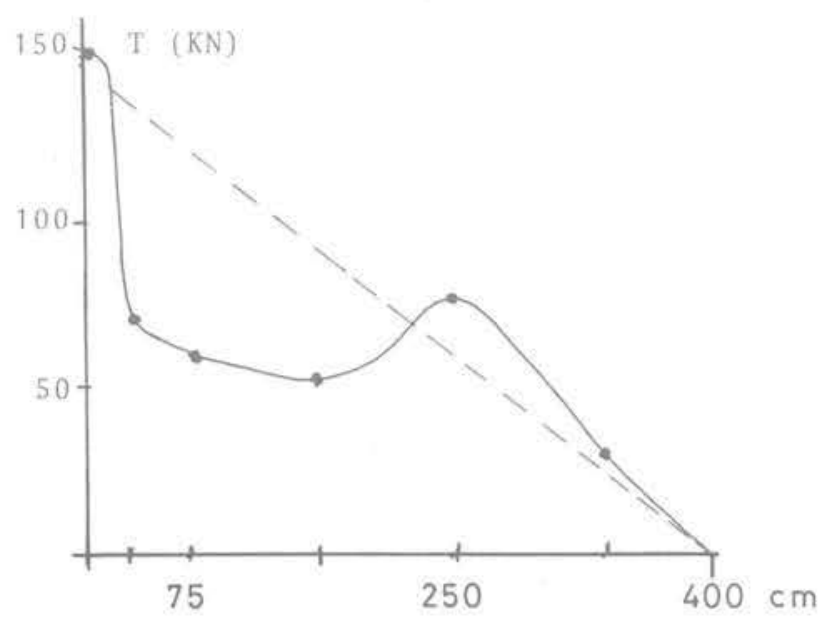

Fig. 8. - Mise en tension à $150 \mathrm{KN}$ d'un boulon passif en clé $d^{\prime}$ un tunnel de $14 m$ d'ouverture dans les marnes. Répartition des efforts (mesures par jauges dynamométriques) KOUMENTAKOS (1977). 
Une méconnaissance, voire une surestimation des capacités de reprise des efforts par les inclusions risque d'entraîner un sous-dimensionnement du clouage pouvant conduire, dans certains cas, jusqu'à la rupture de l'édifice ainsi réalisé par ce procédé.

Dans le cas de réalisations pratiques, un minimum de mesures de contrôle est par ailleurs requis, de manière à vérifier que les déplacements restent inférieurs aux limites fixées par le projeteur. Ce type d'auscultation est généralement réalisé dans la pratique.

\section{QUELQUES APPLICATIONS RÉCENTES}

Des applications intéressantes ont été réalisées au cours des dernières années, notamment en France. A ce titre, il convient de signaler en particulier les expériences relatives aux projets suivants :

- l'exécution en variante de la stabilisation de la fouille de la tour CB2 à la Défense, sur une hauteur de $20 \mathrm{~m}$, dans des remblais, sables de Beauchamp et marnes et caillasses à tendance marneuse. La stabilisation a été assurée à l'avancement par des ancrages de 6 à $8 \mathrm{~m}$, de $25 \mathrm{~mm}$ de diamètre, et une peau de $20 \mathrm{~cm}$ de béton projeté armé de treillis ;

- la réalisation de la rénovation du cours d'Herbouville à Lyon après un glissement de terrain ayant détruit plusieurs immeubles, le 31 juillet 1977 faisant plusieurs morts. Les figures 9 et 10 montrent en coupe la nature des travaux réalisés ainsi que la phase d'avancement de ces derniers en juin 1984.

-210 NGF $m$

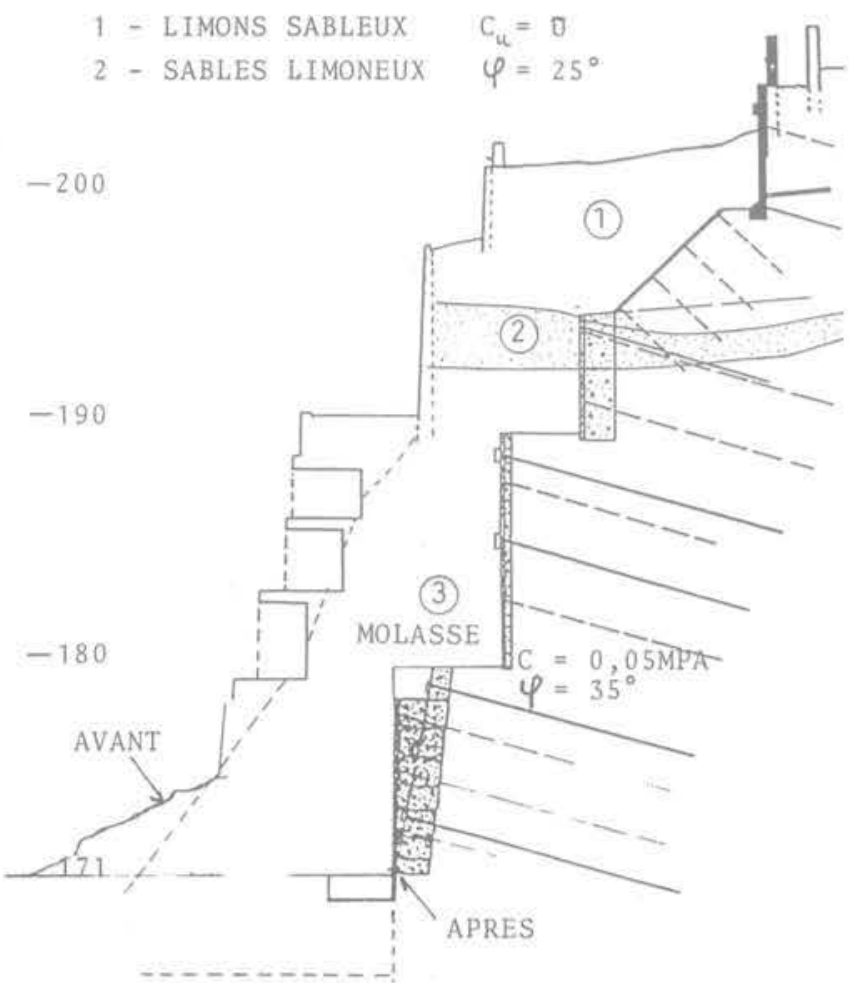

Fig. 9. - Versant du cours d'Herbouville à Lyon avant et après stabilisation.

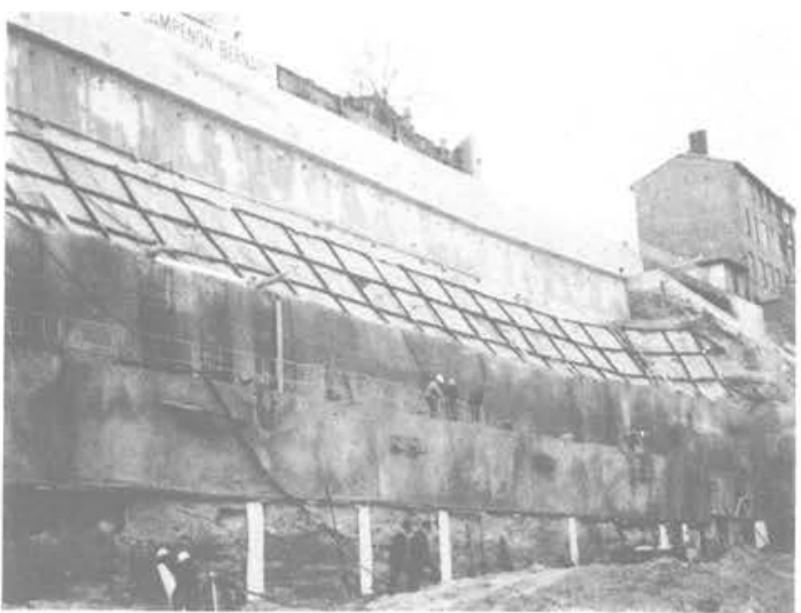

Fig. 10. - Vue du chantier du cours d'Herbouville à Lyon en juillet 1984.

Il convient enfin de signaler, dans les améliorations technologiques, les progrès réalisés dans le domaine du vibrofonçage d'armatures dans les sols instables (qui nécessiteraient normalement un tubage préalable lors de la mise en place de l'armature). L'injection du coulis de scellement (fig. 11) peut avoir lieu pendant ou après le vibrofonçage par un canal axial prévu à cet effet. Avec injection pendant le vibrofonçage, on réalise ainsi un JET BOLT pour lequel le traitement de la zone périphérique de l'inclusion contribue à accroître considérablement la capacité de résistance de l'ensemble. Les paramètres de vibrofonçage sont enregistrés à l'avancement et constituent ainsi une reconnaissance complémentaire des terrains permettant un dimensionnement à l'avancement plus précis du clouage.

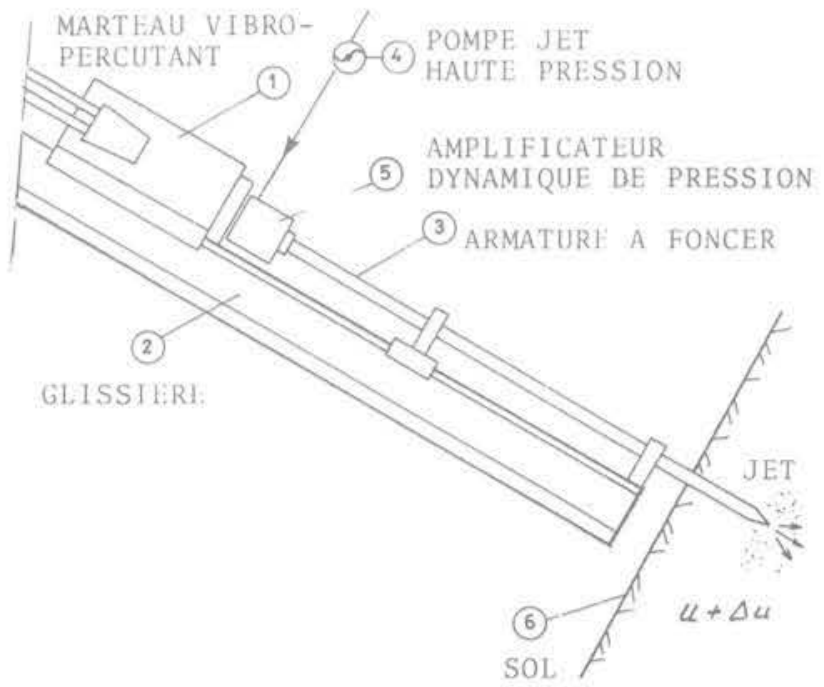

Fig. 11. - Technique CLOU JET.

Vibrofoncage d'armatures avec ou sans injection à l'avancement (" JET BOL TING»). 
Deux exemples parmi tant d'autres de préfabrication de peau de confinement sont donnés à titre complémentaire :

Le premier est constitué de panneaux métalliques préfabriqués assemblés et enrobés de béton coulé en place in situ (stabilisation du talus argileux des Eparris, autoroute A41 à Annecy illustré par la figure 12).

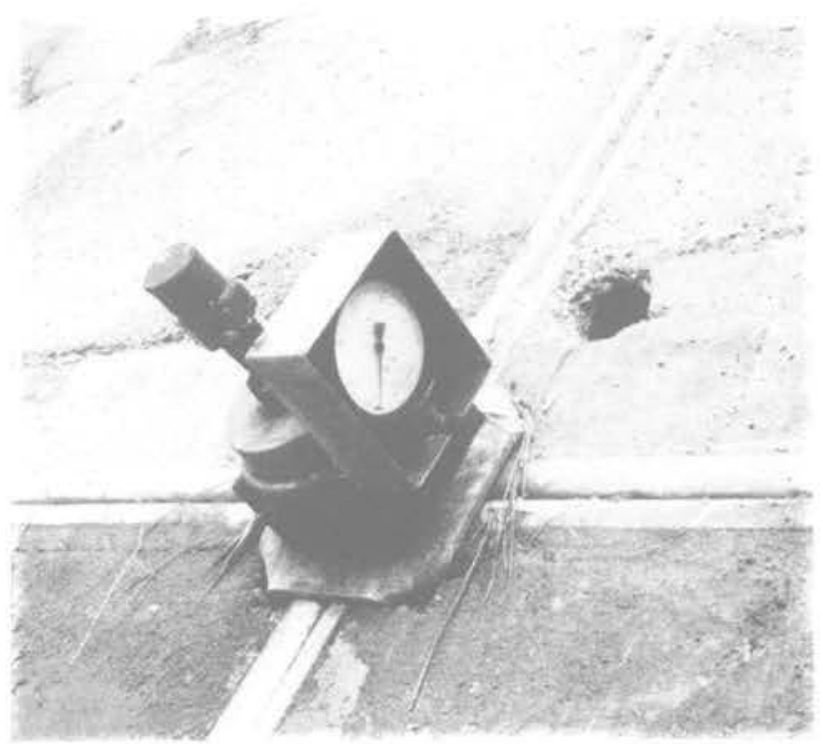

Fig. 12. - Stabilisation d'un talus argileux par panneaux métalliques assemblés et enrobés de béton. Autoroute A41 vers Annecy (Savoie).

Le second concerne la réalisation d'un clouage par éléments de béton préfabriqués et clous vibrofoncés pour la stabilisation d'un versant argileux à Tarare dans le Rhône (fig. 13).

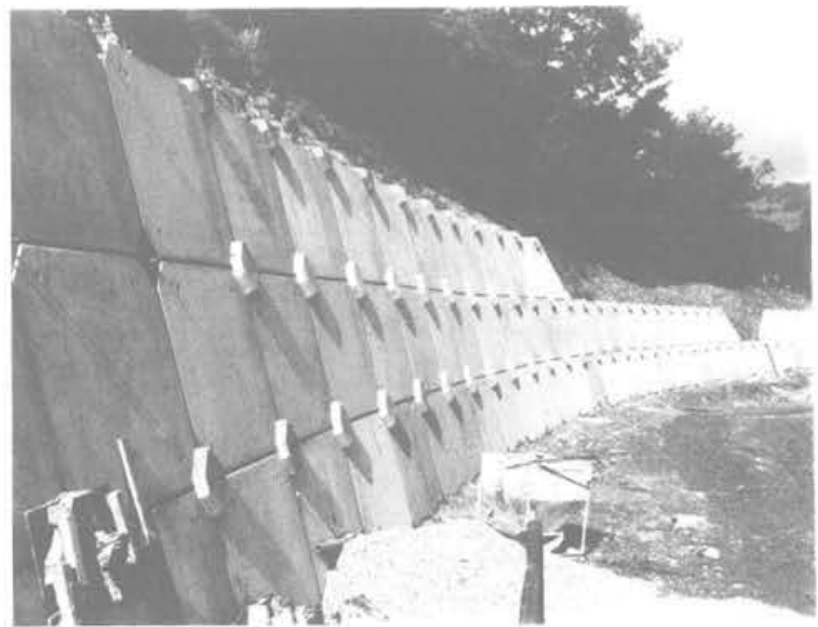

Fig. 13. - Stabilisation d'un déblai à Tarare (Rhône) par panneaux béton préfabriqués cloués au terrain (limons argileux).
Une grande variété de systèmes d'éléments préfabriqués de géométries unique ou complémentaire a été mise au point pour répondre à des critères d'esthétique, de pérennité, voire d'économie. Ces éléments peuvent résoudre à la demande des problèmes spécifiques, comme par exemple de drainage, d'acoustique, d'environnement, etc. La figure 14 montre quelques exemples de tels panneaux.

Les systèmes de JET BOLTING et d'éléments préfabriqués ont fait l'objet de brevets récents.
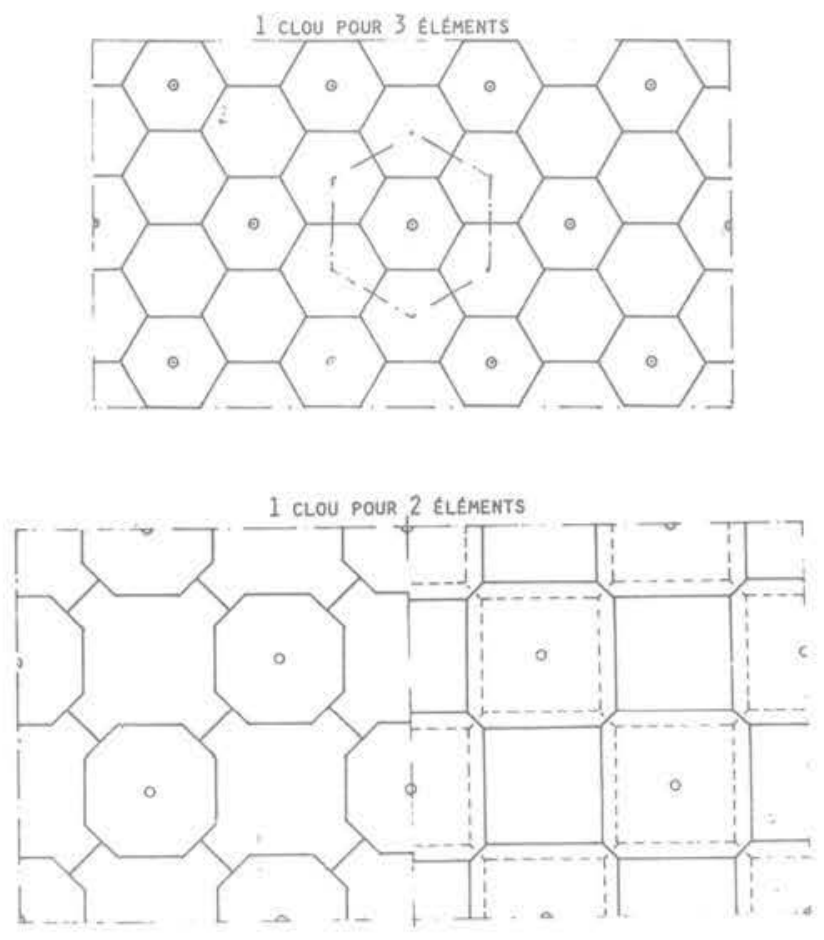

Fig. 14. - Exemples d'éléments préfabriqués pour revêtements de talus.

\section{CONCLUSION}

Le clouage des sols s'est notablement développé au cours des dernières années pour le cas des sols meubles, cohérents, voire granulaires. Le renforcement du sol est surtout apporté par les armatures scellées dans le milieu, solidaires d'une peau de confinement à la surface de l'excavation (talus, puits, ou cavités souterraines).

Les armatures, généralement métalliques, sont mises en œuvre dès les premières phases de l'excavation avec leur géométrie finale, alors que la perturbation des contraintes est encore minimum. Elles sont à l'origine passives et deviennent actives (en travaillant en traction-cisaillement) au fur et à mesure que l'excavation se poursuit. Le rôle stabilisateur de telles armatures est capital dans le processus, il n'a pas été néanmoins clairement mis en évidence ni par la théorie, ni par la pratique, faute de mesures suffisantes (méthodes de dimensionnement insatisfaisantes). 
L'action des armatures, qui s'oppose à toute déformation inadmissible du terrain, est avantageusement complétée par le revêtement de surface (peau de confinement) qui doit rester souple dans son comportement. Ce revêtement est lui aussi mis en œuvre à l'avancement au fur et à mesure de la poursuite de l'excavation.

Cette méthode empirique a connu, au cours des dix dernières années, des applications très intéressantes avec utilisation du béton projeté et d'armatures métalliques scellées à la résine ou au mortier. Des extensions sont possibles avec utilisation de panneaux préfabriqués en surface, soit pour limiter les coûts, soit pour certains objectifs fonctionnels particuliers (aspect esthétique, propriétés phoniques, etc.). La mise en place des armatures peut se faire par forages mais également par vibrofonçage avec injection simultanée (JET BOLTING). Cette dernière technique est particulièrement performante dans les sols pulvérulents instables à toute foration. Le coulis du jet sert à la fois de fluide de lançage, de produit de scellement ultérieur et pour la consolidation du terrain par diffusion latérale.

Cette méthode, qui s'apparente autant à la nouvelle méthode autrichienne de construction des tunnels (utilisant béton projeté et boulonnage pour le soutènement) qu'à la technique de terre armée pour les sols en remblai, connaîtra certainement à l'avenir des applications intéressantes tant :

- par le caractère rationnel des principes mis en œuvre. Elle tire en effet profit au maximum des caractéristiques mécaniques initiales du terrain en utilisant ce dernier comme matériau de construction;

- que sur le plan économique en comparaison avec d'autres méthodes de soutènement.

\section{BIBLIOGRAPHIE}

CARTIER G, et GIGAN J.P. (1982), Experiments and observations on soil nailing structures, VIII E.C. M.S.F.E. d'Helsinki, octobre 1982.

GAESSLER G. (1977), Large scale dunamic test of in situ reinforced earth, Proc. D.M.S.R. 77 Karlsruhe, septembre 1977, vol. II, pp. 333-342.

GAESSLER G., GUDEHUS G. (1981), Soil Nailing Some aspects of a new technique, X, I.C.S.M.F.E., vol. III, Stockholm, pp. 665-670.
GAESSLER G., GUDEHUS G. (1982), Soil Nailing -Statistical Design, VIII E.C. S.M.F.E. d'Helsinki, Proc., vol. II, Sess. 5, pp. 491-494.

GAESSLER G. (1984), Bodenvernagelung, thèse de Dr. Ing., Université de Karlsruhe.

GOULESCO M. et MEDIO J.M. (1981), Soutènement des sols en déblai à l'aide d'une paroi hurpinoise, Revue T.O.S., A.F.T.E.S. n 47, pp. 205-214.

GOULESCO N. (1984), Évolution de la technique de la hurpinoise.

GUILLOUX A. (1984), Évaluation du frottement sol Inclusion dans le clouage des sols, E.N.P.C., Coll. Int., Renforcement des sols et des couches, Paris, 9.11 octobre 1984.

JEWELL R.A. (1980), Some effects of reinforcements on the mechanical behaviour of soil, Cambridge Université, p. 307.

JURAN I. (1977), Dimensionnement interne des ouvrages en terre armée, thèse Dr. Ing., Paris.

JURAN I. (1985), Reinforcement soil systems, A.S.C.E. Geotech. Eng., vol XVI, pp. 39-82.

JURAN I., SCHLOSSER F,, LOUIS C., KERNOA M., ECKMAN B. (1981), Le renforcement des sols par barres passives, X I.C.S.M.F.E., vol. III, Stockholm, 1981, pp. 713-716

KOUMENTAKOS S. (1977), Comportement et rôle du boulon dans les méthodes modernes de soutène. ment, thèse de Dr. Ing., Université de Nancy I, $1^{\text {er }}$ mars 1977.

LOUIS C. (1981), Nouvelle méthode de soutènement des sols en déblais, Revue Travaux, numéro spécial mars 1981 , pp. 67-75.

RABEJAC et al. (1974), Construction d'un mur de soutènement entre Versailles chantiers et Versailles Matelots, Revue générale des Chemins de fer, avril 1974, pp. 232-237.

SCHLOSSER F., JURAN I. (1979), Design Parame. ters of artificially improved soils, General Report VII E.C.S.M.F.E., Brighton, pp. 199-225

SCHLOSSER F., JURAN 1. (1981), Le frottement dans le renforcement des sols, Revue Française de Géotechnique n ${ }^{\circ}$ 16, pp. 65-77.

SHEN C.K. et al. (1981), An in situ earth reinforcement lateral support system. University of California, Devis-Report 81.03 for the National Science Foundation, $188 \mathrm{p}$.

STOCKER M.F. (1976), Bodenvernagelung, D.B.G.T. Nurnberg, D.G.E.G., pp. 639-652.

STOCKER M.F. et al. (1979), Soil Nailing, Coll. Int. Renforcement des sols, Paris, pp. 469-474.

VERRIER G. et MERLETTE P. (1981), Confortement des remblais ferroviaires. Une technique particulière : le clouage, numéro spécial Revue Travaux, mars 1981, pp. $76-81$. 